\title{
Total ozone time series analysis: a neural network model approach
}

\author{
B. M. Monge Sanz and N. J. Medrano Marqués \\ Electronics Design Group - GDE-, Faculty of Sciences, University of Zaragoza, E-50009 Zaragoza, Spain \\ Received: 15 June 2004 - Revised: 19 November 2004 - Accepted: 14 December 2004 - Published: 16 December 2004 \\ Part of Special Issue "Nonlinear analysis of multivariate geoscientific data - advanced methods, theory and application"
}

\begin{abstract}
This work is focused on the application of neural network based models to the analysis of total ozone (TO) time series. Processes that affect total ozone are extremely non linear, especially at the considered European mid-latitudes. Artificial neural networks (ANNs) are intrinsically non-linear systems, hence they are expected to cope with TO series better than classical statistics do. Moreover, neural networks do not assume the stationarity of the data series so they are also able to follow time-changing situations among the implicated variables. These two features turn NNs into a promising tool to catch the interactions between atmospheric variables, and therefore to extract as much information as possible from the available data in order to make, for example, time series reconstructions or future predictions. Models based on NNs have also proved to be very suitable for the treatment of missing values within the data series. In this paper we present several models based on neural networks to fill the missing periods of data within a total ozone time series, and models able to reconstruct the data series. The results released by the ANNs have been compared with those obtained by using classical statistics methods, and better accuracy has been achieved with the non linear ANNs techniques. Different network structures and training strategies have been tested depending on the specific task to be accomplished.
\end{abstract}

\section{Introduction to neural networks}

Since the 1980s neural networks have been applied to a wide range of subjects, most of them mainly within the Economics and Medical fields. The forecasting of bankruptcy situations (Martín del Brío, 1993), the calculation of possible peaks in energy consumption (Alsayegh, 2003; Martín del Brío et al., 1995), or diagnosis of tumoural tissues (Vega-Corona et al., 2003; Zhou et al., 2002), are some of the successful applica-

Correspondence to: B. M. Monge Sanz

(bmonge@unizar.es) tions in these areas. Computer interfaces for speech recognition (Medrano and Martín del Brío, 2000), signal processing (Lajusticia et al., 2003), or users identification (Mitchell, 1997) are other fields in which neural systems are being applied.

Neural networks today are also little by little showing their abilities to solve different problems in Geophysics, several authors have recently applied these systems for classification (Macías et al., 2001), data retrieval (Acciani et al., 2003; Müller et al., 2001), forecasting (Olsson et al., 2004), downscaling (Trigo and Palutikof, 1999), parameterisation (Chevallier et al., 2000) and problems related to the quality of the data series (Reusch and Alley, 2002).

This first section gives a brief description of neural networks (NNs), explains basic terminology and performance algorithms, and also examines the main features that make this kind of system ideal for geophysical applications.

\subsection{What are neural networks?}

An artificial neural network is a multiprocessor computer system based on the parallel architecture of the brain. Each network consists of several simple processors called neurons, or cells, which are highly interconnected and are arranged in several layers (Fig. 1). The first and last layers are the input and the output layer, respectively; and between the input and the output there may be several hidden layers. To every connection between neurons a certain corresponding weight indicates the relevance that an output will have as an input for the next neuron. The interaction between neurons is an adaptive interaction based on the adaptability of the weights' value. In fact, a neural network works by adapting these weights in order to minimize the difference between the response given by the output layer and the real expected value ("target value") for a certain pattern of inputs. The way these weights adapt is called the "training rule", or "learning rule", of the network. So, a neural network has to "learn" in order to work properly, just as our brains do; and it is this fact that 


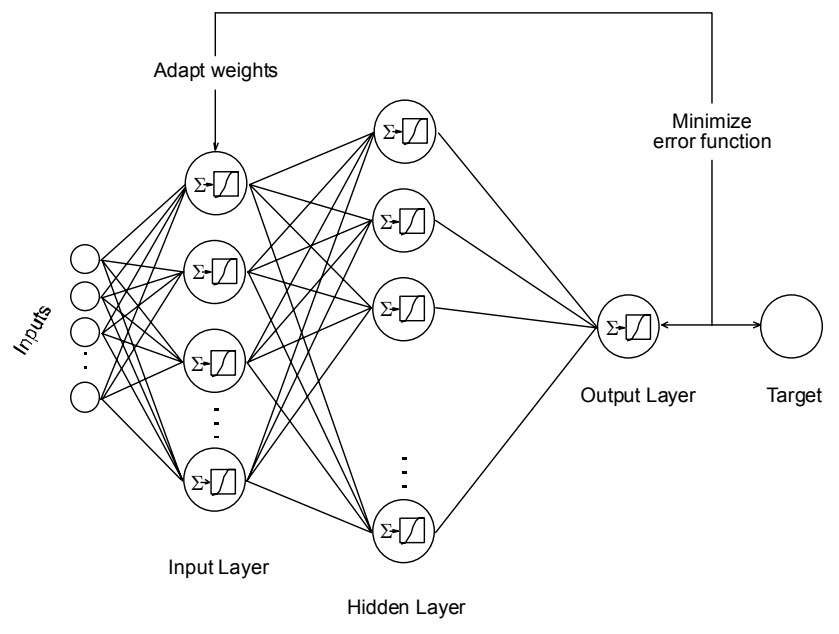

Fig. 1. General scheme of an artificial neural network.

enlarges enormously the fields of application of these artificial neural schemes.

NNs are very helpful systems when numerous examples of the behaviour intended to model are available, since the more examples one has, the simpler the structure of the network can be in order to achieve the same accuracy (Rojas, 1996). It is during the training stage that the $\mathrm{NN}$ learns how to model the behaviour/s under study. There are two main types of training: supervised and unsupervised. It is the first one that we have used and whose fundamentals are explained next.

To train the network, a "training set" of patterns is used. For the supervised learning rules, this set is made up of input vectors, each of them with an associated output target (for the unsupervised rules the set consists of inputs only). The components of the input vectors are the values of the "predictors", or independent variables, and the targets are the values of the "predictand", or dependent variable, for the corresponding input components. For every pattern of the training set, the net releases a certain response, this response is then compared with the corresponding target value, and the error between both quantities is calculated. The error function is then used to adjust the connection weights in order to minimize the difference between net outputs and targets. There are many different methods of setting the weights ("training algorithms"). We have chosen the Levenberg-Marquardt algorithm (Hagan and Mohammad, 1994), which, with respect to the more extended backpropagation algorithm, is faster and more reliable (see next section).

Like what happens in our brains, new interconnections can be established if the learning process requires it (due for example to the fact that a certain behaviour is observed more frequently). Once the NN has been trained, a set of predictors with unknown values for the dependent variable, can be presented to the NN, and then the responses given by the network are expected to have an accuracy within the range reached during the training process. The precision that the system must be able to achieve is one of the parameters that can be modified by the designer, taking into account certain rules regarding number of patterns and size of the network.

\subsection{Advantages for geophysics}

Neural networks present several characteristics that make them ideal systems for dealing with atmospheric and climatological data. First of all, NNs are non linear systems, which makes them an ideal tool for catching non-linearities. Secondly, they are highly versatile systems which can easily adapt to circumstances, making them able to pick up temporal variations, while classical statistics assume the stationarity of the series. NNs are a very useful phenomenological approach when the dynamics of the problem is either not known or is too complex. In addition, because of the high interconnectivity that a NN presents, this kind of model is very tolerant to errors or noise in the input data. Therefore, NNs are robust and flexible systems which are able to deal with non-linear and non-stationary series. To ensure a better tracking of time variations in the relationships between variables, different strategies can be followed: moving window training set, bootstrapping techniques, ...

In our case we have applied NNs models for the treatment of TO time series. As for almost every climate or weather time series, we have a long available record of data, and we have to deal with extremely non-linear relationships. Solar activity, the chemical composition of the atmosphere, wind regime, or stratospheric intrusions are some of the highly non-linear processes that affect the total amount of ozone. In addition, since the relationships between variables are expected to vary over time, we were interested in a method which is not only able to cope with non-linearities, but also able to track time-changing situations. These reasons led us to consider NNs as the best potential candidate to solve the problem.

Besides, one major advantage of neural nets is that no special requirements need to be fulfilled a priori by the time series. In general the more complex the problem, the larger the number of data required, but also the size and architecture of the network play a fundamental role. There is no fixed criteria to say that the ideal length of the time series should be one or another, simply because there is no fixed criteria to use one type of neural network or another, nor one training algorithm or another. Neural networks are flexible enough to be applied to any time series, provided that some points are taken into consideration. Primarily, the overtraining of the network must be always avoided. Overtraining means that the net is not able to generalize any rules for patterns different from those used for training because it has learned (memorized) the patterns in the training set during the learning stage.

Several studies about which should be the ideal size of a network in order to avoid overtraining (Baum, 1989; Haykin, 1999), have supplied a rule that relates the number of patterns used for training and the maximum number of weights that the network can have. Such a rule is: $p=\mu / w$, where $p$ is the order of the number of patterns, $w$ is the total number of weights and $\mu$ is the order of the precision one wants to 
reach. In our applications this rule has been taken into account to minimise the risk of having an overfitted network.

The next section offers a brief presentation of the data and the methodology we have used, including the NNs configurations and the chosen training algorithm. In Sect. 3, one model based on neural networks is presented for the substitution of missing values within the data series and compared with classical statistical techniques. Section 4 deals with time series reconstruction.

\section{Data and method}

For this work we have chosen TO series corresponding to stations located at European mid-latitudes, which, because of their geographical position, are strongly influenced by atmospheric dynamics. All TO data have been retrieved from the World Ozone and Ultraviolet Radiation Data Centre (WOUDC). The time series used are made up of total ozone mean monthly values for the following locations (Fig. 2): Lisbon $\left(38.8^{\circ} \mathrm{N}, 9.1^{\circ} \mathrm{W}\right)$ in Portugal, Arosa $\left(46.8^{\circ} \mathrm{N}, 9.7^{\circ} \mathrm{E}\right)$ in Switzerland, and Vigna di Valle $\left(42.18^{\circ} \mathrm{N}, 12.2^{\circ} \mathrm{E}\right)$ in Italy.

The artificial NNs we have employed are feedforward configurations of the multilayer perceptron (MLP) implemented with Matlab software. Feedforward networks are nets in which signals flow from the input to the output neurons, in a forward direction. There also exist recurrent or feedback networks which have closed-loop paths, from a unit back to itself or to units from a previous layer (Fausset, 1994). Feedforward networks have been proved to be able to approximate a function with a finite number of discontinuities to any degree of accuracy (Principe et al., 2000).

One of the most extended training algorithms for MLP structures is backpropagation (Rumelhart et al., 1986). This method basically consists of a gradient descent technique based on the Widrow-Hoff rule. However, the algorithm chosen to train our ANN models was the Levenberg-Marquardt algorithm (L-M) because, for moderate seized networks, it presents important advantages that help to overcome two of the main problems that arise from backpropagation training: the Levenberg-Marquardt can converge more rapidly, and the risk of the final weights becoming trapped in a local minimum is much lower. On the other hand, the L-M algorithm requires more computational memory (Matlab, 1997), since it assesses second derivatives.

Concrete applications of these neural network configurations to ozone data series and the results obtained will be discussed next.

\section{NNs models for missing data treatment}

When dealing with time series, some kind of treatment for the missing data is essential since most of the analysis methods cannot be performed otherwise. Classical methods such as substitution of the gaps by the mean value of the series, or

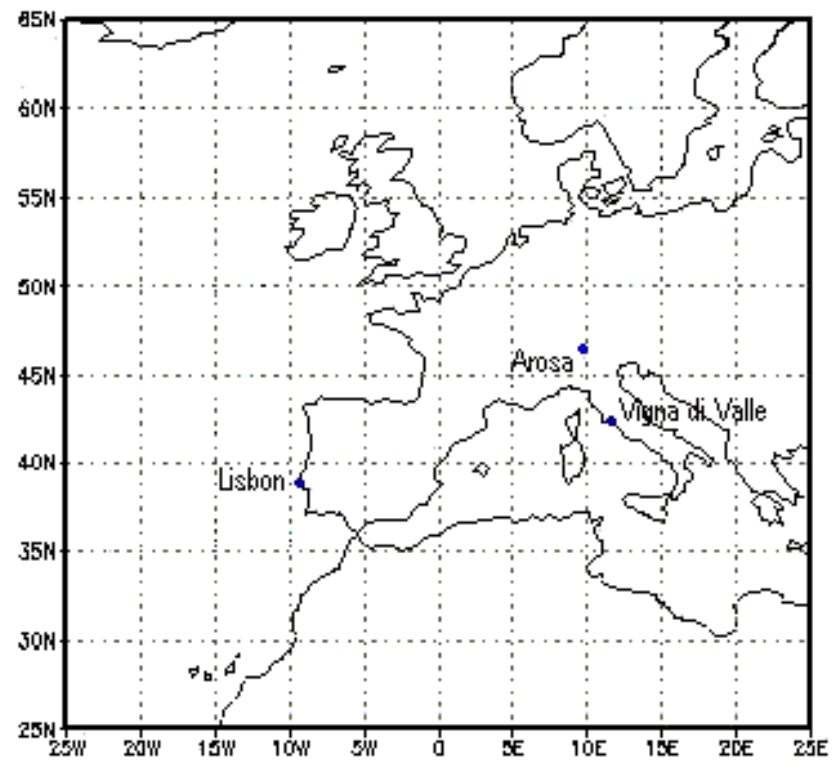

Fig. 2. Map of the stations whose data series have been analysed.

interpolation from the nearest neighbours, persistence techniques or arbitrary value substitution, are unable to catch time variations, or dependence with other variables variations. We propose here the alternative method of using nonlinear neural networks to improve missing values substitution.

In order to design the most appropriate network model and training strategy we have distinguished between isolated missing values or long gaps within the data series. In this work we describe how the isolated data within TO series have been filled by using a NN model, and compare the results with those obtained by applying a linear interpolation technique.

For the estimation of the isolated gaps within the data series, the $n$ previous and the $n$ following values to the missing one are presented as inputs to the network. And then the net releases the searched value. The structure of the network used for this interpolation model is a pyramidal $(2 n 2 n-11)$ configuration. For the single output neuron the lineal transfer function is used, while for the other layers the log-sigmoid function has been chosen. Data series were standardized before being processed by the net.

Best results are obtained for $n=2$, compared with those obtained for $n=1, n=3, n=4$. The physical reason is quite probably that the number of data considered with $n=2$ is 5 $(2+2$ predictors and the missing one), thus almost one semiperiod of the annual ozone signal component is being taken into account. TO series, at the latitudes considered here, present a seasonal periodicity of 12 months, high ozone season for spring-summer and low-ozone season for autumnwinter. So taking fewer values we were not assuming variations within an interval affecting the searched value, and with more predictors we were including seasonal variations which must be avoided for the neural network to be able to detect 
Table 1. RMSE values for the results obtained with the non-linear model based on neural networks and with the lineal model, for the three considered data series.

\begin{tabular}{ccc}
\hline & NN non-linear model & Linear model \\
\hline station & RMSE & RMSE \\
\hline Lisbon & 0.79 & 7.62 \\
Vigna di Valle & 2.64 & 3.23 \\
Arosa & 2.89 & 2.99 \\
\hline
\end{tabular}

anomalies.

To evaluate the model performance, simple statistical tests have been applied: the Root-Mean-Square-Error (RMSE) and the explained variance (EV); which also permit an easy comparison with the results obtained with other methods. In our case, the response given by the NN model has been compared to the one achieved using a linear interpolation technique. By using a perceptron with linear activation function that has been trained with the LMS method or Widrow-Hoff rule, it is possible to implement a linear interpolation function by way of a simple NN model. This model generates the same results as a linear regression (Widrow and Winter, 1988; Trigo and Palutikof, 1999).

The series of Lisbon for the period June 1967-July 1975 is shown in Fig. 3a. The first part of the dataset, i.e. June 1967-November 1973, was used to train the network, while the set December 1973-July 1975 has been used to validate the model. The validation period is indicated by an arrow. In the graph, the real values are represented by stars and the circles stand for the values released by our NNs based model. The second diagram (Fig. 3b) is the result for the same series and the same period of time but using a linear interpolation model. It can be seen that the performance of the first model is much better: stars and circles match up better for the nonlinear model. In fact, the RMSE for the NN non-linear model is $0.79 \%$ whereas for the linear model it is $7.62 \%$. Results for all the three stations are summarized in Table 1. It is shown that the NN non-linear model improves the results obtained with a linear regression model.

In order to provide further proof of the non-linearity of the model, we include a histogram with the distribution of the weights values for the input layer and the hidden layer (Fig. 4) of the network used in this application. The meaning of the weights in a neural system is not directly comparable with the coefficients in a classical regression model, nevertheless, as can be seen from the chart, most of the weights have an absolute value higher than 5, with several above 20 . Such a weights distribution points out that the network works far from the linear range of the sigmoidal activation function, so that we can assure that the nonlinear part of the network plays the main role in the model, and that the model we are dealing with is clearly non linear.

In this section the substitution of isolated missing values has been analysed. The estimation of longer gaps requires
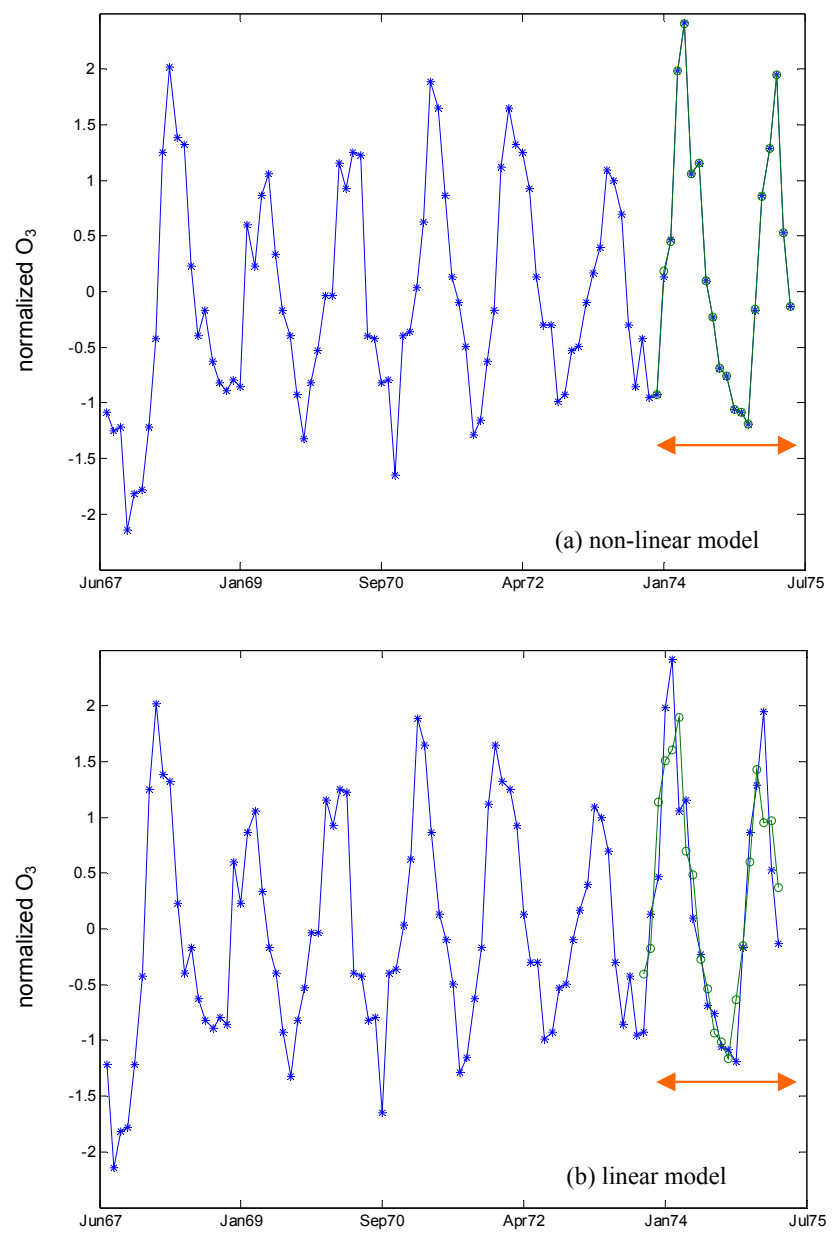

Fig. 3. Real values $(*)$ and modelled values (o) for the ozone series of Lisbon during the period June 1967-July 1975. Results for the interval December 1973-July 1975 have been obtained with a non-linear model based on neural networks (a), and with a linear regression model (b). The better performance of the NNs model can be seen.

more complex and specific methods, therefore NNs are also a promising alternative to accomplish the task. In MongeSanz and Medrano-Marqués (2003), non-linear models based on NNs that use the North Atlantic Oscillation Index as a predictor have been successfully applied for the assessment of long missing periods of data.

\section{NNs for time series reconstruction}

In the previous section NNs have been used to discover the absent values inside a data series. A more ambitious application is the reconstruction of a series beyond the available data record, either forwards or backwards. For such a purpose we have developed a NN model which is able to extend the considered ozone series when a longer one is used as predictor. The example shown here is the forward extension of the series of Vigna di Valle by using the series of Arosa. The Arosa register has been chosen as a good pre- 


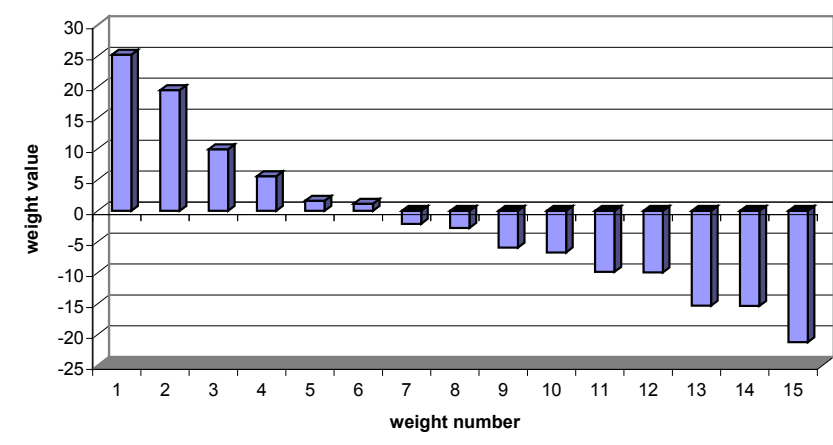

Fig. 4. Weights distribution of the neural network used for the interpolation of isolated missing data. The values shown correspond to the input layer and to the hidden layer of the net. Since most of them are high values, the network is acting far from the linear range of the sigmoidal function.

dictor because of the high correlation that it presents with the Italian one, and because it is the longest European total ozone record, with observations in this alpine location dating back to the 1920's. Moreover, the time series of Arosa is not only the longest but also one of the best quality registers of TO because of the revisions and updates that have been made to ensure adaptation between different instrumentation and measurement strategies to provide a homogeneous series (Staehelin et al., 1998). The length and quality of the Arosa register make it the ideal series to predict values for shorter or more incomplete series which are correlated enough.

To find the TO value at Vigna for a certain month, which we call the current month, we use the following set of predictors: the $\mathrm{n}$ previous values to the considered month of the Vigna seires, the $\mathrm{n}$ previous of the Arosa series, and the current month value of Arosa. From these inputs the net provides the mean value of TO for the current month at Vigna. For the case analysed here the set of data is June 1967-October 1980, and the period November 1975-October 1980 has been reserved for validation.

The model structure used consists of a two layer MLP with $k$ input neurons and one cell at the output layer. These models ( $k+1$ models) have been used before for different meteorological applications, see for instance (Trigo and Palutikof, 1999); with these models better results are achieved for our application than with more complex structures. For the output layer the linear transfer function has been chosen, whilst the quality of the results seems to be independent from the election of the log-sigmoid or the hyperbolic tangent transfer function for the input layer.

For this particular application we have experimented with a technique that is of high interest at the moment because it improves the performance of individual neural networks: as the final signal of our model, we have considered the average of the output of several $k+1$ nets. Since we average the results given by several of these $k+1$ individual networks, the parameter being $k=1, \ldots, 2 n+1$ (one $k$ value for one network), we are using a "neural network ensemble". The neural network ensembles technique is a learning scheme where

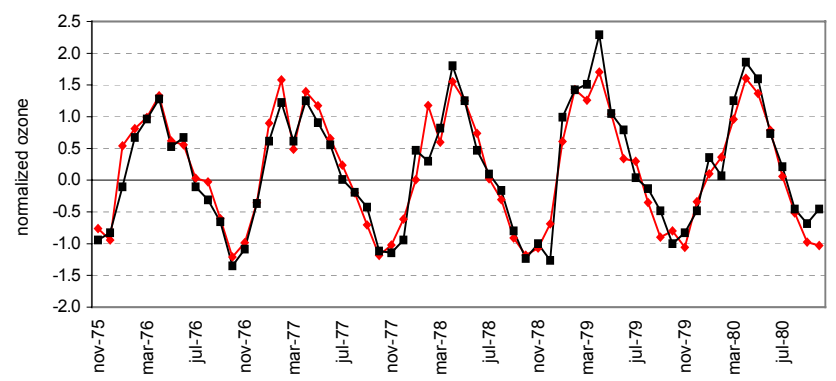

Fig. 5. One month ahead reconstruction for Vigna di Valle by way of the NNs ensemble $k+1$ for $n=1$ (red line), and the observed real series at the station from November 1975 to October 1980 (black line).

a finite number of individual networks is trained to solve a problem.

A neural network ensemble consists of a set of different individual neural networks, trained with the same or different predictors, in order to give the same output variable. The output of the ensemble is a combination of the outputs of the individual NNs. It has been shown that the generalization capability of a NN can be improved by using this kind of ensemble learning (Sharkey, 1999). The way the individual predictions are combined strongly depends on the application the ensemble is used for. For regression tasks, as is our case, the individual outputs can be averaged (Opitz and Shavlik 1996) or weight averaged (Perrone and Cooper 1993); while for classification majority voting may be an ideal combining approach (Hansen and Salamon, 1990).

The ensemble we have used is made up of a collection of neural networks, each of these networks with a different number $k$ of input cells. For a given number $n$ of values of the Vigna series, the ensemble contains $2 n+1$ networks, every network with its corresponding $k$, and the parameter $k$ takes values from 1 to $2 n+1$. In our application, every net in the ensemble provides the mean value of TO. By taking the average of all these responses (output of the ensemble) as the final signal of our model, we get an answer with a lower variance.

The method of the neural network ensembles has however a substantial drawback: ensembles are more difficult to interpret than single networks, although some works have already addressed the problem of the extraction of rules from these structures, see for instance (Zhou et al., 2003).

In Fig. 5 the results obtained with the model $k=1$, and with $n=1$, are represented together with the real signal for the time interval under consideration. The high explained variance value $(E V>88.0 \%)$ means that the shape of the original signal is accurately reproduced by the model.

Compared with the application in Sect. 3, where isolated gaps were filled up by using the previous and following values of the series itself, here we have also included Arosa as a predictor variable. Just to highlight the improvement achieved by including Arosa comparative tests have been carried out. Table 2 shows the results obtained when the recon- 
Table 2. RMSE and EV values for the forward reconstruction of Vigna series, comparing the inclusion of Arosa data as an input variable with the results obtained using the Vigna series only.

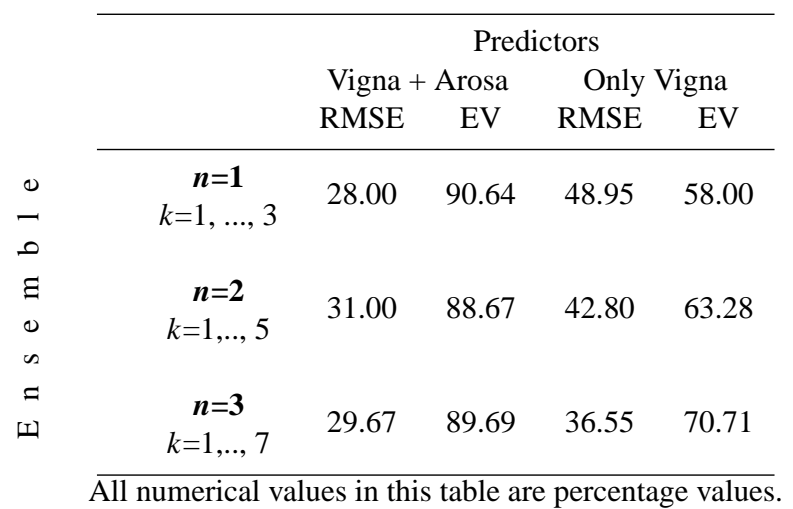

struction of the Vigna series is tried using only data from this series, and the results attained with the inclusion of Arosa data in the model.

As can be deduced from Table 2, better results are obtained when Arosa is included as a predictor, lower RMSE and higher correlation are achieved. In addition, the spread in the RMSE and EV values is a good indicator for the robustness of the strategy: when we use only Vigna the results seem to be much more dependent on the number of inputs and the particular ensemble used.

In general, the inclusion of related variables as predictors for the model will always improve the quality of the results. Although it is true that one must consider whether such inclusions offer a sufficiently worthwhile improvement for the computational cost they imply. In cases like ours, where both the size of the network and the number of input variables are rather small, the benefits of including a second variable, here Arosa series, compensate by far for the minimal increase in the computing time of the model, which still remains within the order of $10 \mathrm{~s}$.

Besides, we are passing from having just one predictor series (Vigna) to having two predictor series (Arosa and Vigna), in a case like this, with any kind of model, not just with neural network schemes, one must expect more benefits than problems.

Considering again the results in Fig. 5, one can state that, given the high correlation between real and modelled series and the ability of this NNs structure to predict the right sign of the TO variations, it is shown that the model including Arosa, without any modification, is suitable for forecasting the sign of TO monthly anomalies. However, if we want to improve accuracy for the exact values we should include new predictors, such as for example the Arctic Oscillation Index, which exhibits very high correlations with TO series throughout the whole year over these European areas (Staehelin et al., 2002; Monge-Sanz et al., 2003).

\section{Future work and concluding remarks}

Because of their great versatility and capability of dealing with non-linear and non stationary series, neural network systems are the ideal approach for geoscience data treatment. In this work, several models based on NNs for the analysis of TO time series have been presented for different applications concerning missing data treatment and time series reconstruction. Such models have proved to achieve better results than some other classical techniques. In addition, the simplicity of our NNs based models allows us to consider further applications without the risk of involving schemes which are too complex or time consuming. Some of these applications involve the backwards-reconstruction of time series into pre-instrumental periods, or the forecasting of the magnitude under analysis. Different time-scales can also be considered just by choosing the appropriate set of predictors.

Acknowledgements. The authors are grateful to the World Ozone and Ultraviolet Radiation Data Centre (WOUDC) for providing ozone data, and to the Climatic Research Unit (CRU) of the University of East Anglia for the NAO Index data series. Our special thanks go to A. F. Pacheco for his continuous support and advice during the realization of this work, and his encouragement to develop it further. We also thank the anonymous referees for their constructive remarks and suggestions which have greatly helped to improve the quality of this paper.

Edited by: M. Thiel

Reviwed by: two referees

\section{References}

Acciani, G., D’Orazio, A., Delmedico, V., De Sario, M., Gramegna, T., Petruzzelli, V. and Prudenzano, F.: Radiometric profiling of temperature using algorithm based on neural networks, Electron. Lett., 39, 17, 1261-1263, 2003.

Alsayegh, O. A.: Short-term load forecasting using seasonal artificial neural networks, In. J. Power Energy Sys., 23, 3, 137-142, 2003.

Baum, E. B.: A proposal for more powerful learning algorithms, Neural Computation, 1, 295-311, 1989.

Chevallier, F., Morcrette, J.-J., Cheruy, F., and Scott, N. A.: Use of a neural-network-based longwave radiative transfer scheme in the ECMWF atmospheric model, Quart. J. Roy. Meteor. Soc., 126, 761-776, 2000.

Fausset, L.: Fundamentals of Neural Networks. Architectures, Algorithms and Applications, Prentice Hall International Editions, New Jersey, 1994.

Hagan, M. and Mohammad, B.: Training Feedforward Networks with the Marquardt Algorithm, IEEE Transactions on Neural Networks, 5, 6, 989-993, 1994.

Hansen, L. K. and Salamon, P.: Neural network ensembles, IEEE Transactions on Pattern Analysis and Machine Intelligence, 12, 993-1001, 1990.

Haykin, S.: Neural networks, A comprehensive foundation, 2nd edition, Prentice-Hall, 1999.

Lajusticia, C., Medrano, N., Zatorre, G., and Martín, B.:AnalogDigital Multipliers with Non-Ideal Behaviour in Neural Network Circuits, in: Proceedings of the XVIII Conference on Design 
of Circuits and Integrated Systems, DCIS 2003, Ciudad Real (Spain), 2003.

Macías Macías, M., López Aligué, F., Serrano Pérez, A., and Astilleros Vivas, A.: A comparative study of two neural models for cloud screening of Iberian Peninsula Meteosat images, edited by Mira, J., Springer Verlag, Berlin Heidelberg, IWANN 2001, LNCS 2085, 184-191, 2001.

Martín del Brío, B., Medrano, N., Ramírez, I., Domínguez, J.A., Barquillas, J., Blasco, J., and García, J.: Short-term electric power load forecasting using artificial neural networks. Part I: self-organizing networks for classification of day-types, 14th IASTED Int. Conf. On Modelling, Identification and Control, Igsl, Austria, 1995.

Martín del Brío, B. and Serrano Cinca, C.: Self-organizing neural networks for análisis and representions of data: Some financial cases, Neural Computing and Applications, 1, 193-206, 1993.

MATLAB Reference Guide: Neural Networks Toolbox User's Guide, 1997.

Medrano, N. and Martín del Brío, B.: Computer voice interface using the mouse port, XVI Design of Integrated Circuits and Systems Conference, DCIS 2000, Montpellier, France, 2000.

Mitchell, T.: Machine Learning, McGraw Hill, 1997.

Monge-Sanz, B. and Medrano Marqués, N.: Artificial Neural Networks Applications for Total Ozone Time Series, edited by Miram, J., Springer Verlag, Berlin Heidelberg, IWANN 2003, LNCS 2687, 806-813, 2003.

Monge-Sanz, B. M., Casale, G. R., Palmieri, S., and Siani, A. M.: Ozone Loss over South Western Europe, Its Relation with Circulation and Transport, Air Pollution Research Report 79, Stratospheric Ozone 2002, European Commission, Belgium, 396-399, 2003.

Müller, M. D., Kaifel, A. K., Weber, M., and Tellmann, S.: Realtime total ozone and ozone profiles retrieved from GOME data using neural networks, in Proceedings 2001 EUMETSAT meteorological Satellite Data User's Conference, Antalya, EUMETSAT, Darmstadt, Germany, 2001.

Olsson, J., Uvo, C. B., Jinno, K., Kawamura, A., Nishiyama, K., Koreeda, N., Nakashima, T., and Morita, O.: Neural Networks for Rainfall Forecasting by Atmospheric Downscaling, J. Hydr. Engin., 9, 1, 1-12, 2004.

Opitz, D. W. and Shavlik, J. W.: Actively searching for an effective neural network ensemble, Connection Science, 8, 337-353, 1996.
Perrone M. P. and Cooper, L. N.: When networks disagree: Ensemble method for neural networks. Artificial Neural Networks for Speech and Vision, edited by Mammone, R. J., Chapman \& Hall, New York, 126-142, 1993.

Principe, J., Euliano, N., and Lefebvre, W.: Neural and Adaptive Systems, Wiley, 2000.

Reusch, D. B. and Alley, R. B.: Automatic weather stations and artificial neural networks: improving the instrumental record in West Antarctica, Monthly Weather Review, 130, 12, 3037-3053, 2002.

Rojas, R.: Neural Networks, a Systematic Introduction, SpringerVerlag, 1996.

Rumelhart, D. E., Hinton, G. E., and McClelland, J. L.: A General Framework for Parallel distributed Processing, Parallel Distributed Processing, edited by Rumelhart, D. E. and McClelland, J. L., Foundations, 1, 1986.

Sharkey, A. J. C.: Combining Artificial Neural Nets: Ensemble and Modular Multi-Net Systems, Springer-Verlag, 1999.

Staehelin, J., Mäder, J., Weiss, A. K., and Appenzeller, C.: Longterm ozone trends in Northern mid-latitudes with special emphasis on the contribution of changes in dynamics, Phys. Chem. Earth, 27, 461-469, 2002.

Staehelin, J., Renaud, A., Bader, J., McPeters, R., Viatte, P., Hoegger, B., Bugnion, V., Giroud, M., and Schill, H.: Total ozone series at Arosa (Switzerland): Homogeneization and data comparison. J. Geophys. Res., 103, D5, 5827-5841, 1998.

Trigo, R. M. and Palutikof, J. P.: Simulation of daily temperatures for climate change scenarios over Portugal: a neural network approach, Climate Res., 13, 45-59, 1999.

Vega-Corona, A., Álvarez-Vellisco, A., and Andina, D.: Feature vectors generation for detection of microcalcifications in digitized mammography using neural networks, edited by Mira, J., Springer Verlag, Berlin Heidelberg, IWANN 2003, LNCS 2687, 583-590, 2003.

Widrow, B. and Winter, R.: Neural nets for adaptive filtering and adaptive pattern recognition, IEEE Computer, 1988.

Zhou, Z., Jiang, Y., and Chen S.: Extracting rules from trained neural networks ensembles, AI Communications, 16, 1, 3-15, 2003.

Zhou, Z., Jiang, Y., Yang, Y., and Chen S.: Lung cancer cell identification based on artificial neural network ensembles, Artificial Intelligence in Medicine, 24, 1, 25-36, 2002. 